CONF-900923--3

UCRL- JC-104991

PREPRINT

\title{
Inner-Shell Ionization of Lithium-Like Chromium Ions
}

\author{
D. A. Vogel \\ P. Beiersdorfer \\ R. E. Marrs \\ K. L. Wong \\ R. Zasadzinski \\ High Temperature Physics Division \\ Prepared for the Submittal to \\ Zeitschrift f. Physik D \\ $\mathrm{V}^{\text {th }}$ International Conference on \\ the Physics of Highly-Charged Ions. \\ Giessen, FRG
}

September 10-14, 1990

September 7, 1990

This is a preprint of a paper intended for publication in a journal or proceedings. Sirice changes may be made before publication, this preprint is made available with the understanding that it will not be cited or reproduced without the permission of the author.
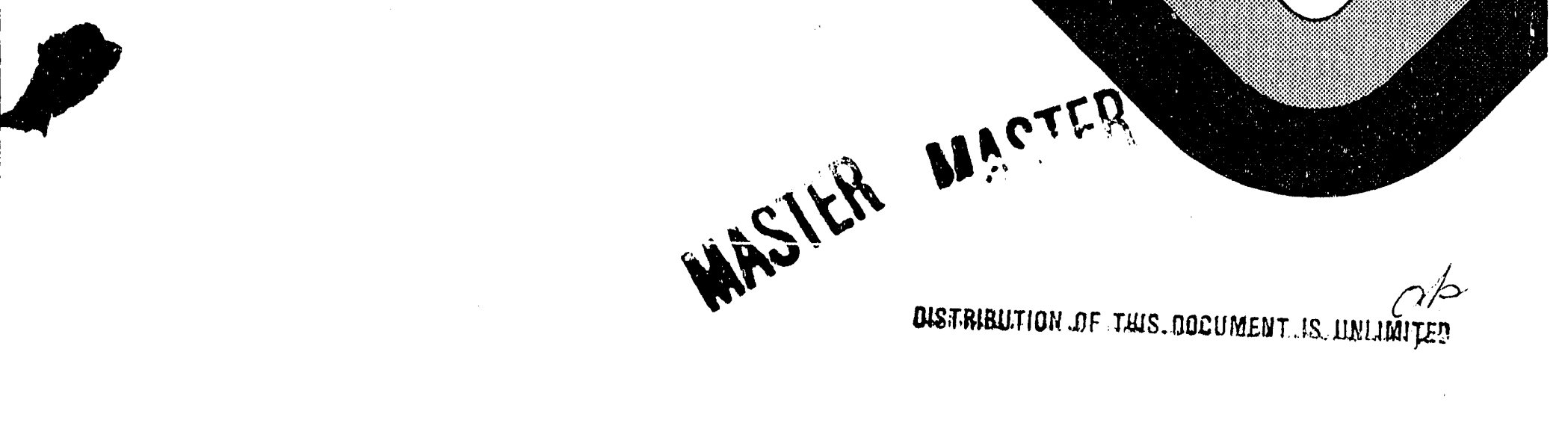

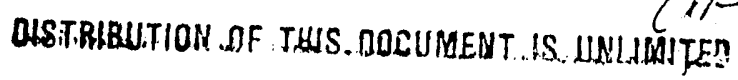




\section{DISCIAINER}

This document was prepared as an account of work sponsored by an agency of the linited States Government. Neither the linited States Government nor the Eniversity of California nor any of their employees, makes any warranty. express or implied. or assumes any legal liahility or responsibility for the accuracy. completeness, or usefulness of any information, apparatus. product, or process disclosed, or represents that its use would not infringe privately owned pights. Reference herein to any specific commercial priducts, process, or service by trade name, trademark, manufacturer, or otherwise, does not necessarily constitute or imply its endorsement, recommendation. or favoring by the linifed Sitales (ionernment or the l niversily of California. The viess and opinions of authors espressed herein do not necessarily state or reflect those of the I niled States Government or the l'niversity of California, and shall not be used for advertising or product endorsement purposses.

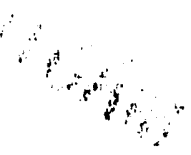


UCRL-JC- -104991

DE9 1001749

\title{
INNER-SHELL IONIZATION OF LITHIUM-LIKE CHROMIUM IONS
}

\author{
D. Vogel, P. Beiersdorfer, R. Marrs, K. Wong, R. Zasadzinski \\ High Temperature Physics Division \\ Universty of California, Lawrence Livermore National Laboratory, \\ Livermore, $C A 94550$
}

\begin{abstract}
We have used high-resolution $x$-ray spectroscopy to investigate inner-shell ionization of $\mathrm{Cr}^{21}+$ ions by electron impact using the Electron Beam Ion Trap at Lawrence Livermure Laboratory. Our measuremerits indicate that inner-shell ionization enhances the intensity of the radiative transition $1 \mathrm{~s} 2 \mathrm{~s}{ }^{3} \mathrm{~S}_{1} \rightarrow 1 \mathrm{~s}^{2}{ }^{1} \mathrm{~S}_{0}$.
\end{abstract}




\section{Introduction}

Helium-like ions occur in many types of plasma. Examples include solar flares and tokamaks, as well as plasmas produced by intense laser pulses. Since the ions in a plasma repeatedly undergo excitation by colliding electrons, they continually emit $x$-rays, most of which escape the plasma. These impact excitation $x$-rays provide important clues to the composition, charge balance, density and temperature of the plasma. The helium-like $\mathrm{x}$-ray spectrum is of particular interest because satellite lines from neighboring lithium-like and beryllium-like charge states mingle with the He-like lines. Their relative intensities therefore provide a convenient indication of the charge balance. Processes such as dielectronic recombination and inner-shell ionization complicate the picture, however, because they populate excited levels in adjacent charge states.

In hot ionizing plasmas, inner-shell ionization can have a profound effect on the intensity of the transition. $1 \mathrm{~s} 2 \mathrm{~s}^{3} \mathrm{~S}_{1} \rightarrow 1 \mathrm{~s}^{2}{ }^{1} \mathrm{~S}_{0}$. It is precisely the $\mathrm{x}$-rays resulting from this transition that allow us to isolate the inner-shell process. The Electron Beam Ion Trap (EBIT) has already been used to investigate both impact excitation and dielectronic recombination [1,2]; now we have studied ionization interactions as well (Wong et al. [3] have investigated outer-shell ionization of $\mathrm{Ba}^{53+}$ ions).

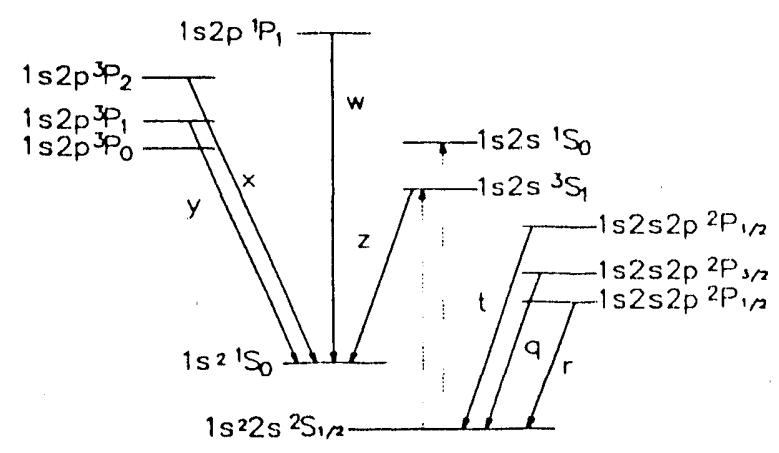

Fig. $1 \mathrm{He}$-like and Li-like $\mathrm{n}=2$ levels (energy axis is not drawn to scale). The $2 \rightarrow 1 \mathrm{x}$-ray lines observed in our experiment are labeled using the notation of Gabriel [4]. The three doubly excited Li-like levels shown are the only ones with appreciable cross sections for direct excitation. The vertical dotted lines indicate inner-shell ionization.

\section{Theory}

Figure 1 shows the energy levels involved (He-like and Li-like), as well as their radiative decays to the ground state. The resonance line $w$, an electric dipole (E1) transition, is the strongest. Next in intensity is $y$, also an E1 transition, but weaker than w because it is an intercombination line. The other intercombination line, $x$, is a magnetic 
quadrupole transition. Line $\mathrm{z}$, a magnetic dipole transition, is a forbidden line, but in EBIT, even $z$ is strong because the plasma density is low. Neither the ${ }^{3} \mathrm{P}_{0}$ nor the ${ }^{1} \mathrm{~S}_{0}$ level decays radiatively to the ground state since $j=0$ to $j=0$ transitions are strictly forbidden (the latter state does decay through two-photon emission, while nuclear hyperfine splitting enables the $1 \mathrm{~s} 2 \mathrm{p}{ }^{3} \mathrm{P}_{0} \rightarrow 1 \mathrm{~s}^{2}$ transition for the ${ }^{53} \mathrm{Cr}$ nucleus, whose abundance is about $9.5 \%$ ). The satellite lines $\mathrm{q}, \mathrm{r}$, and $\mathrm{t}$, come from doubly excited Li-like ions. Such doubly-excited ions can be produced by dielectronic recombination; these three levels also have significant cross sections for direct (inner-shell) excitation frorn the Li-like ground state.

Line $z$ can be enhanced strongly by inner-shell ionizaiion. Inner-shell ionization of a $\mathrm{Li}$-like ion removes one of the two K-shell electrons, e.g.,:

$$
\mathrm{Cr}^{21+}+\mathrm{e}^{-}+\rightarrow\left(\mathrm{Cr}^{22+}\right)^{*}+2 \mathrm{e}^{-}
$$

leaving the resulting He-like ion in either the $1 \mathrm{~s} 2 \mathrm{~s} \quad \mathrm{~S}_{1}$ or $1 \mathrm{~s} 2 \mathrm{~s} \mathrm{~S}_{0}$ level. The threshold for this process is $7.4 \mathrm{keV}$, much higher than the (outer-shell) ionization potential of $\mathrm{Cr}^{21+}$, and close to the ionization potential of $\mathrm{Cr}^{22+}$. A possibility also exists (if the electron energy is slightly greater than $7.4 \mathrm{keV}$ ) that the ion ends up in one of the other He-like levels, a process known as shake-up. The ${ }^{3} \mathrm{~S}_{1}$ level, being the lowest, is also fed by cascades. Cascades from the ${ }^{3} \mathrm{P}_{0}$ and ${ }^{3} \mathrm{P}_{2}$ levels, as well as states with $\mathrm{n}>2$ provide about 80 percent of $z$ 's intensity.

At the low electron and ion densities present in EBIT, collisional de-excitation is rare and metastables decay before they can undergo additional excitations. Hence, line intensities can be expressed in terms of the ground state density and excitation cross sections [5]. The photon emission rate for line $w$ is given by:

$$
\mathrm{R}_{\mathrm{w}}=\mathrm{je}_{\mathrm{e}} \mathrm{N}_{\mathrm{He}_{\mathrm{c}}} \sigma_{\mathrm{w}}
$$

where $\mathrm{j}_{\mathrm{c}}$ is the electron current density, $\mathrm{N}_{\mathrm{Hc}}$ is the number of ground-state $\mathrm{He}$-like ions in the beam, and $\sigma_{\mathrm{w}}$ is the cross section for electron impact excitation. For $\mathrm{z}$, we must add terms for cascades and inner-shell ionization:

$$
\mathrm{R}_{\mathrm{z}}=\mathrm{N}_{\mathrm{HCj}} \mathrm{j}_{\mathrm{c}}\left(\sigma_{\mathrm{z}}+\sum_{\mathrm{i}} \sigma_{\mathrm{i}} \mathrm{B}_{\mathrm{iz}}\right)+\mathrm{N}_{\mathrm{Li}} \mathrm{j}_{\mathrm{c}} \sigma_{\mathrm{ii}}
$$

The second term is a sum over all the states $i$ that can feed $z$, where $\sigma_{i}$ is the state's excitation cross section and $\mathrm{B}_{\mathrm{i} z}$ is the branching ratio. The last term gives the inner-shell contribution; it is the only one that depends on $\mathrm{N}_{\mathrm{Li}}$, the Li-like ion density. The observed emission intensity is proportional to the photon rates:

$$
I_{i}=R_{i} \cdot G
$$


where the constant $G$ depends on the geometry of the detector and the EBIT. Since we cannot measure the ion number densities $\mathrm{N}_{\mathrm{He}}$ and $\mathrm{N}_{\mathrm{Li}}$, we take the intensity ratio:

$$
\frac{\mathrm{I}_{\mathrm{z}}}{\mathrm{I}_{\mathrm{w}}}=\frac{\mathrm{R}_{\mathrm{z}}}{\mathrm{R}_{\mathrm{w}}}=\frac{\sigma_{\mathrm{z}}}{\sigma_{\mathrm{w}}}+\frac{\mathrm{N}_{\mathrm{Li}}}{\mathrm{N}_{\mathrm{He}}} \frac{\sigma_{\mathrm{ii}}}{\sigma_{\mathrm{w}}}
$$

where $\sigma_{\mathrm{z}}{ }^{\prime}=\sigma_{\mathrm{z}}+\Sigma \sigma_{\mathrm{i}} \mathrm{B}_{\mathrm{iz}}$. To determine $\mathrm{N}_{\mathrm{Li}} / \mathrm{N}_{\mathrm{He}}$, we can use one of the Li-like satellites:

$$
\frac{\mathrm{I}_{q}}{\mathrm{I}_{\mathrm{w}}}=\frac{\mathrm{N}_{\mathrm{Li}}}{\mathrm{N}_{\mathrm{He}}} \frac{\sigma_{\mathrm{q}}}{\sigma_{\mathrm{w}}}
$$

where $\mathrm{I}_{\mathrm{q}}$ and $\sigma_{\mathrm{q}}$ correspond to line q. Combining (5) and (6) gives a linear relationship among $I_{z}, I_{q}$, and $I_{w}$ :

$$
\mathrm{I}_{\mathrm{z}}=\frac{\sigma_{\mathrm{z}}^{\prime}}{\sigma_{\mathrm{w}}} \mathrm{I}_{\mathrm{w}}+\frac{\sigma_{\mathrm{ii}}}{\sigma_{\mathrm{q}}} \mathrm{I}_{\mathrm{q}}
$$

Thus, by measuring the line intensities at different charge states, one can determine $\sigma_{\mathrm{ii}} / \sigma_{\mathrm{q}}$.

\section{Experimental Procedure}

Ws used the Livermore Electron Beam Ion Trap (EBIT), described by Levine et al. [6], to generate highly charged chromium ions. By adjusting the beam accelerating voltage, one can probe electron-ion interactions at energies from $4-25 \mathrm{keV}$. At energies above the inner-shell threshold, $\mathrm{Cr}^{22+}$ ions predominate overwhelmingly. To increase the fraction of $\mathrm{Cr}^{21+}$ ions, we alternate the electron beam energy between the KLL dielectronic resonance, at about $4.2 \mathrm{kV}$, and the observation energy $(9-23 \mathrm{kV})$. Since the DR cross section is large, many $\mathrm{Cr}^{22+}$ ions recombine into the Li-like ground state. The DR resonance has a gaussian shape, so slightly changing the lower level voltage reduces the $\mathrm{Cr}^{21+} / \mathrm{Cr}^{22+}$ ratio. Typical dwell times are $5 \mathrm{~ms}$ for observation and $10 \mathrm{~ms}$ at the $\mathrm{KLL}$

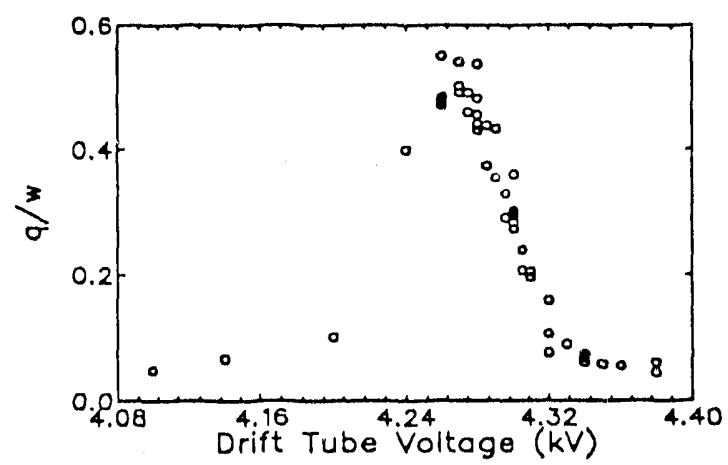

Fig. 2. Line ratio $I_{q} / I_{w}$ as a function of drift tube voltage shows how scanning over KLL resonance adjusts the Li-like fraction. 
resonance. Figure 2 shows the relative charge balance, indicated by the ratio $I_{q} / I_{w}$, as a function of beam energy near the DR resonance.

\section{Results}

We recorded the $x$-ray lines using a von Hámos crystal spectrometer [7] set up to view at a 90-degree angle to the electron beam. We were able to observe inner-shell ionization at six different interaction energies, ranging from $8.8 \mathrm{keV}$ to $23.5 \mathrm{keV}$. Figure 3 shows the effect of inner-shell ionization in the $20.3-\mathrm{keV}$ spectrum. Compared to line $\mathrm{w}$, satellite lines $\mathrm{q}$ and $\mathrm{r}$ are larger because more $\mathrm{Cr}^{21+}$ is present; at the same time, $\mathrm{z}$ has been noticeably enhanced.


Fig. 3 High-resolution $x$-ray spectra at beam energy of $20.3 \mathrm{keV}(\lambda / \Delta \lambda=1800)$. (a) $\mathrm{Cr}^{21+}$ fraction is small so satellite lines are small and line $z$ is little enhanced. (b) With more $C r^{21+}$ present, $q$ and $r$ are larger; corresponding enhancement of $z$ from inner-shell ionization is evident.

When the charge balance is varied, the line ratios show the expected linear relationship. Figure 4 is a plot of the line ratio $I_{z} / I_{w}$ versus $I_{q} / I_{w}$ for $8.8 \mathrm{keV}$ anc for 20.3 $\mathrm{keV}$, along with a linear fit to each data set, derived using a nonlinear least squares procedure. We find the same relationship between $I_{z} / I_{w}$ and $I_{r} / I_{w}$. Table I summarizes the results for all six interaction energies. Note that the fits to $I_{q}$ and $I_{r}$ give approximately the same intercept in each case. Although the slopes are proportional to $\sigma_{\mathrm{q}}$ and $\sigma_{\mathrm{r}}$ respectively, the intercept should equal $\sigma_{\mathrm{z}}^{\prime} / \sigma_{\mathrm{w}}$ in both cases.

The linear correlation between $\mathrm{I}_{\mathrm{z}} / \mathrm{I}_{\mathrm{w}}$ and $\mathrm{I}_{\mathrm{q}} / \mathrm{I}_{\mathrm{w}}$ in each case makes it evident that inner-shell ionization has a significant effect on $z$. Additional evidence comes from the increase in slope with energy (up to $20.3 \mathrm{keV}$ ) since $\sigma_{\mathrm{ii}}$ is predicted to increase correspondingly. At the same time, the possibility of shake-up cannot be excluded. We also checked for correlations between lines $\mathrm{y}$ and $\mathrm{x}$ and the Li-like satellites. Both showed 


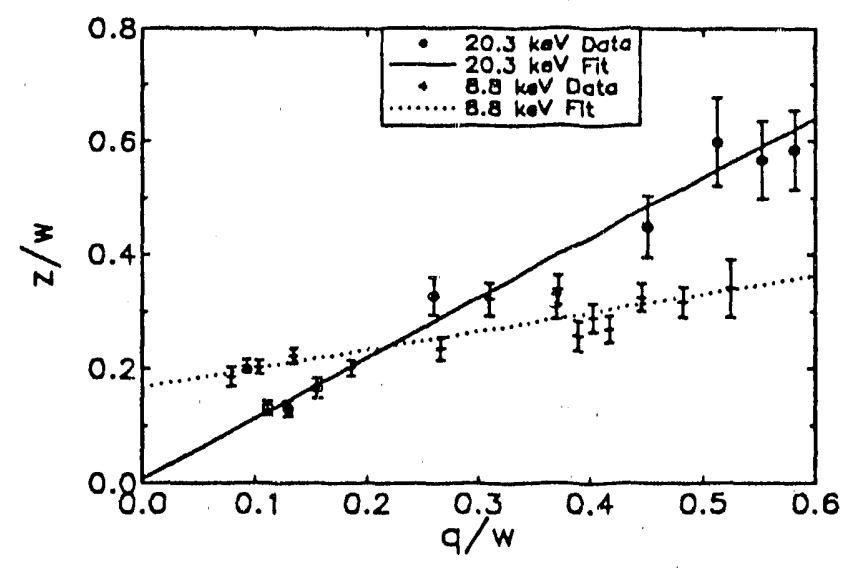

Fig. 4.. $I_{z} / I_{w}$ versus $I_{q} / I_{w}$ for $8.8 \mathrm{keV}$ and $20.3 \mathrm{keV}$. Linear relationships show line enhancement due to inner-shell ionization. Slopes are equal to $\sigma_{\mathrm{ii}} / \sigma_{\mathrm{q}}$.

a slight positive slope at each energy, although in the case of $x$, the value was too small to be conclusive. Line y shows a definite linear correlation, but the slopes do not vary monotonically with energy as with $z$. The enhancement of $y$ may be caused by overlap with line $t$ since these two were not completely resolved by the spectrometer.

Table I. Linear fit parameters for intensity ratio $I_{z} / I_{w}$ versus $I_{q} / I_{w}$ and $I_{q} / I_{w}$ for different energies (in keV)

\begin{tabular}{|rllll|}
\hline \multicolumn{4}{c}{$z / w$ vs. $q / w$} & \multicolumn{2}{c|}{$z / w$ vs. $r / w$} \\
\hline Energy & Slope & Intercept & Slope & Intercep \\
\hline 8.8 & $0.32 \pm .03$ & $0.17 \pm .01$ & $0.92 \pm .11$ & $0.17 \pm .01$ \\
11.8 & $0.56 \pm .06$ & $0.12 \pm .02$ & $1.78 \pm .23$ & $0.11 \pm .02$ \\
14.8 & $0.72 \pm .07$ & $0.06 \pm .02$ & $2.47 \pm .34$ & $0.05 \pm .03$ \\
17.8 & $0.91 \pm .08$ & $0.02 \pm .02$ & $2.99 \pm .37$ & $0.01 \pm .03$ \\
20.3 & $1.10 \pm .07$ & $0.01 \pm .01$ & $2.85 \pm .27$ & $0.00 \pm .02$ \\
23.3 & $1.13 \pm .08$ & $0.03 \pm .01$ & $2.90 \pm .28$ & $0.03 \pm .02$ \\
\hline
\end{tabular}

We would like to thank. E. Magee and D. Nelson for expert technical support throughout the experiment. This work was performed under the auspices of the U. S. Department of Energy by Lawrence Livermore National Laboratory under contract No. W-7405-ENG-48. 


\section{REFERENCES}

1. R.E. Marrs, M.A. Levine, D.A. Knapp, and J.R. Henderson, Phys. Rev. Lett. 60, 1715 (1988).

2. D.A. Knapp, R.E. Marrs, M.A. Levine, C.L. Bennett, M.H. Chen, J.R. Henderson, M.B. Schneider, and J.H. Scofield, Phys. R'v. Lett. 62, 2104 (1989).

3. K. Wong, P. Beiersdorfer, D. Vogel, R. Marrs, and M. Levine (in this volume).

4. A. H. Gabriel, Mın. Not. R. Astron. Soc. 160, 99-119 (1972).

5. J. Dubau and S. Volonte, Rep. Prog. Phys. 43, 199 (1980).

6. M.A. Levine, R.E. Marrs, J.R. Henderson, D.A. Knapp, and M.B. Schneider, Physica Scripta T22, 157 (1988).

7. P. Beiersdorfer, R. Marrs, J. Henderson, D. Knapp, M. Levine, D. Platt, M. Schneider, D. Vogel, and K. Wong, Rev. Sci. Instrum. 61 (1990). 

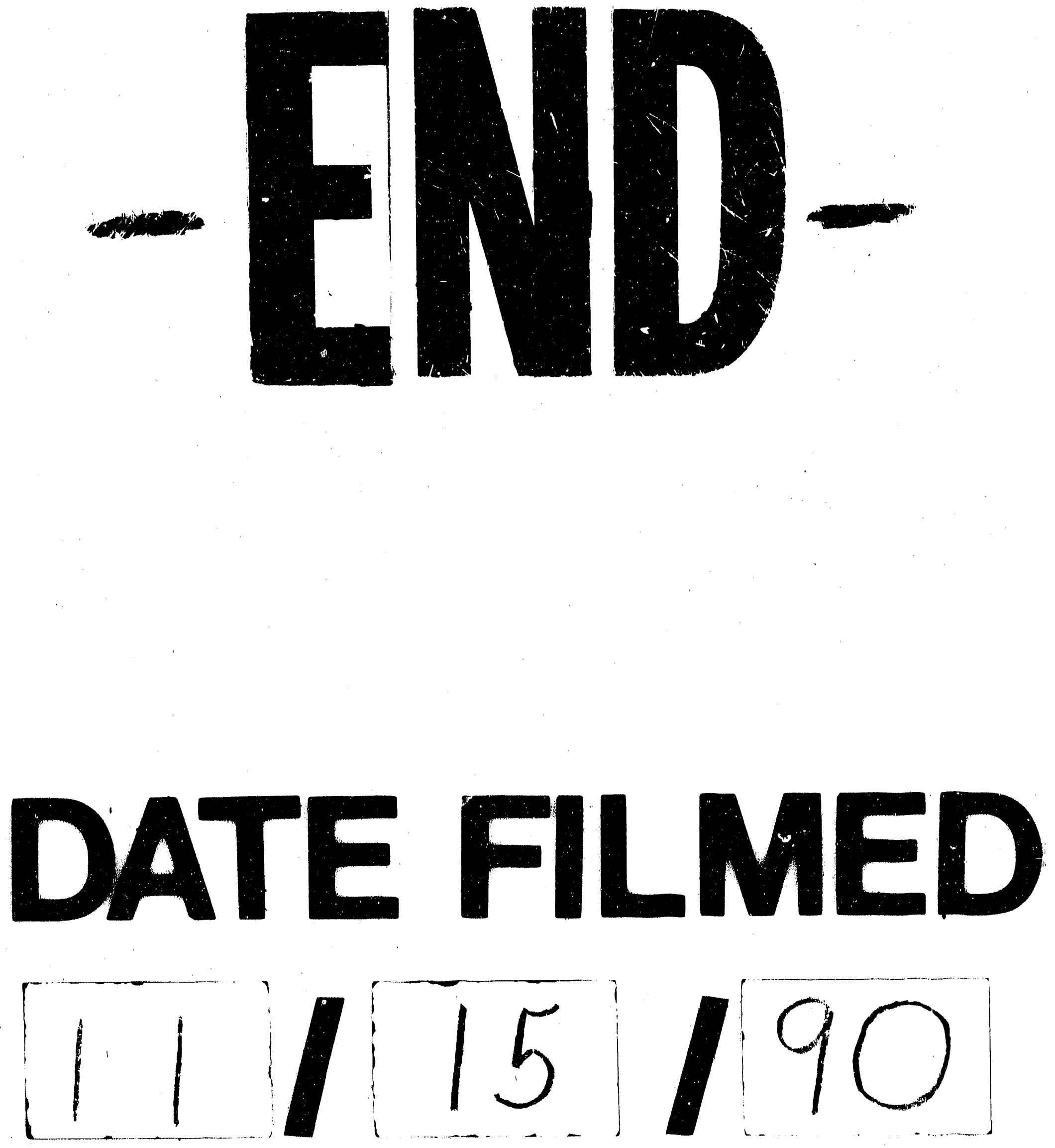
